\title{
The Minimally Invasive Management of Ureteropelvic Junction Obstruction in Horseshoe Kidneys
}

\author{
Costas D. Lallas, M.D., FACS \\ Thomas Jefferson University Hospital \\ Raymond W. Pak, M.D. \\ Thomas Jefferson University Hospital \\ Christopher Pagnani, M.D. \\ Thomas Jefferson University Hospital
}

Scott G. Hubosky, M.D.

Thomas Jefferson University Hospital

Bripbtw thisankeraAditional works at: https://jdc.jefferson.edu/urologyfp Thomas Jefferson University Hospital

Part of the Urology Commons

\section{Let us know how access to this document benefits you See next page for additional authors}

\section{Recommended Citation}

Lallas, M.D., FACS, Costas D.; Pak, M.D., Raymond W.; Pagnani, M.D., Christopher; Hubosky, M.D., Scott G.; Yanke, M.D., Brent V.; Keeley, M.D, Frank X.; and Bagley, M.D., FACS, Demetrius H., "The Minimally Invasive Management of Ureteropelvic Junction Obstruction in Horseshoe Kidneys" (2011). Department of Urology Faculty Papers. Paper 12.

https://jdc.jefferson.edu/urologyfp/12

This Article is brought to you for free and open access by the Jefferson Digital Commons. The Jefferson Digital Commons is a service of Thomas Jefferson University's Center for Teaching and Learning (CTL). The Commons is a showcase for Jefferson books and journals, peer-reviewed scholarly publications, unique historical collections from the University archives, and teaching tools. The Jefferson Digital Commons allows researchers and interested readers anywhere in the world to learn about and keep up to date with Jefferson scholarship. This article has been accepted for inclusion in Department of Urology Faculty Papers by an authorized administrator of the Jefferson Digital Commons. For more information, please contact: JeffersonDigitalCommons@jefferson.edu. 


\section{Authors}

Costas D. Lallas, M.D., FACS; Raymond W. Pak, M.D.; Christopher Pagnani, M.D.; Scott G. Hubosky, M.D.;

Brent V. Yanke, M.D.; Frank X. Keeley, M.D; and Demetrius H. Bagley, M.D., FACS 


\title{
As submitted to:
}

\author{
World Journal of Urology
}

\author{
And later published as
}

\section{The Minimally Invasive Management of Ureteropelvic Junction Obstruction in Horseshoe Kidneys}

\author{
Volume 29, Issue 1, 2011, Pages 91-95
}

\section{DOI: $10.1007 / \mathrm{s} 00345-010-0523-9$}

Costas D. Lallas, M.D., FACS; Raymond W. Pak, M.D.; Christopher Pagnani, M.D.;

Scott G. Hubosky, M.D.; Brent V. Yanke, M.D.; Frank X. Keeley, M.D.; Demetrius H. Bagley, M.D., FACS

Department of Urology, Thomas Jefferson University Hospital, Philadelphia, Pennsylvania

Corresponding author:

Costas D. Lallas, MD, FACS

Assistant Professor of Urology

Kimmel Cancer Center

Thomas Jefferson University Hospital

1025 Walnut Street

Suite 1100 
Philadelphia, Pennsylvania 19107

215-955-6961 office

215-923-1884 fax

Costas.lallas@jefferson.edu 


\begin{abstract}
Purpose: Data regarding the treatment of ureteropelvic junction obstruction (UPJO) in horseshoe kidneys are limited. We performed a retrospective analysis of our experience with minimally-invasive treatment of UPJO in patients with this anomaly.
\end{abstract}

Methods: Between March of 1996 and March 2008, 9 patients with horseshoe kidneys were treated for UPJO at our institution. Of these patients, 6 were managed with retrograde endopyelotomy, 2 with laparoscopic pyeloplasty, and one by robotic pyeloplasty. Outcomes of these procedures were retrospectively reviewed.

Results: A total of nine patients were available for analysis. Four of six patients who underwent endopyelotomy had available follow-up, with a mean of 56 months. The success rate for these patients was $75 \%$. Two of three patients $(67 \%)$ in the laparoscopic/robotic cohort were successfully treated with a mean follow-up of 21 months.

Conclusions: UPJO in horseshoe kidneys can pose a therapeutic dilemma. The minimally-invasive treatment of these patients is feasible with good success rates for both endopyelotomy and laparoscopic/robotic pyeloplasty.

Key words: ureteropelvic junction obstruction, horseshoe kidney, endopyelotomy, pyeloplasty 


\section{Introduction}

Horseshoe kidneys are the most common congenital renal fusion anomaly, estimated to occur in $0.1 \%-0.25 \%$ of births. ${ }^{[1]}$ Although most horseshoe kidneys are asymptomatic, individuals with this anomaly have an incidence of ureteropelvic junction obstruction (UPJO) of $15-33 \% .^{[2]}$ The increased incidence of UPJO may be related to several factors including the high insertion of the ureter into the renal pelvis, the anatomic relation of the ureter to the isthmus, and the highly variable blood supply to the kidney. The development of urolithiasis, which occurs in $21-60 \%$ of patients, as well as associated anatomical abnormalities, which occur in up to $30 \%$ of these patients, may also play a role. ${ }^{[3]}$

The minimally invasive treatment of UPJO in anatomically normal kidneys has been well described. Although endoscopic (retrograde or antegrade) correction of UPJO has been associated with lower success rates, it remains the least invasive option. Laparoscopic pyeloplasty has proven to be safe and effective when compared to open surgery. ${ }^{[4,5]}$ For this procedure, however, a steep learning curve exists that requires expertise in intracorporeal suturing and tying with laparoscopic instruments. However, the daVinci ® surgical system (Intuitive Surgical, Sunnyvale, CA) has enabled these laparoscopic techniques to be performed with greater ease, shorter operative times, and equivalent results. ${ }^{[6]}$

The data regarding the outcomes of minimally invasive treatments for UPJO in patients with horseshoe kidney anomalies are limited. Our goal was to compare the outcomes and discuss the technical challenges unique to horseshoe anomalies among the minimally invasive treatment options. 


\section{Patients and Methods}

After institutional IRB approval, a retrospective chart review of patients treated for UPJO was initiated. The charts of 279 patients who underwent endoscopic or laparoscopic correction of UPJO since 1996 to the present were reviewed. The clinical presentation was reviewed for signs and symptoms of UPJO. Incidental findings were recorded as well. All patients underwent pre- and post-operative diuretic renography to establish baseline renal function as well as degree of obstruction. All patients underwent ureteroscopic examination with endoluminal ultrasound. The presence of crossing vessels, high insertion and segment length were recorded. For laparoscopic and robotic cases, endoluminal ultrasound during a diagnostic endoscopic procedure, or CT angiography was obtained to delineate the anatomy of the UPJ and surrounding structures. Patients were followed every 3-6 months for the first year with a diuretic renogram and annually thereafter. When we evaluate our patients using $t^{1} 1 / 2$ criteria success is defined with $t^{1} \frac{2}{2}$ less than 10 minutes (strict success) or 10 to 20 minutes (relative success). When the renogram demonstrates delayed drainage (more than 20 minutes) but shows relative improvement in the $t^{1} / 2$ compared to preoperative values, as long as the patient remains asymptomatic (subjective success) and the split function improves or stays stable, then the repair is also considered patent. Alternatively if the $t^{1 / 2}$ demonstrates relative success (10 to 20 minutes) and the patient has a relapse of symptoms (subjective failure) and a decline in function by diuretic renogram, the repair is considered a failure.

Endopyelotomy was conducted in a retrograde fashion using a flexible ureteroscope with a 1.9 Fr electrode or 365 um laser fiber. The standard laser setting was 
energy of $1.0 \mathrm{~J}$ with a rate of $15 \mathrm{~Hz}$. The UPJ was incised until the presence of fat. The area was then stented with two double pigtail ureteral stents.

Laparoscopic pyeloplasty was conducted with a standard transperitoneal approach utilizing 3 or 4 trocars, and for the robotic pyeloplasty, a 3-arm set-up was utilized with a camera port, two $8-\mathrm{mm}$ robotic ports and an additional 12-mm assistant port for suction and suture passing. An Anderson-Hynes dismembered pyeloplasty was performed in all cases. The isthmus of the horseshoe deformity was not divided. Our technique for this

procedure has been described elsewhere. ${ }^{[7]}$ Because of the similarities between laparoscopic and robotic pyeloplasty, the combined success of these procedures was reported.

\section{Results}

In the endopyelotomy cohort, 4 males and 2 females were treated with a mean age of 43.7 years (range 25-72 years). Obstruction was on the right in 2 patients, and the left in 4 . All 6 of these patients presented with primary UPJO. Of these patients, 2 were noted to have crossing vessels by endoluminal ultrasound, 4 were noted to have high insertion, and 1 had stone disease on the side of obstruction. The average length of stay was one day with no transfusions performed. Four patients had sufficient follow-up (average of 56 months with a range of 10-108). All four of these patients had diuretic renal scans $>6$ months after the procedure. Three patients were successfully treated at last follow-up with no symptoms and unobstructed diuretic renograms (one was indeterminate with a $t^{1} / 2$ of 17 minutes). The one documented failure presented with 
recurrent renal colic and was determined to be obstructed on renogram. The overall success rate was $75 \%$.

In the laparoscopic/robotic cohort, the average age was 57 years. Two of the three patients presented with secondary UPJO (previous endopyelotomy). One was noted to have a crossing vessel at the transition point of the obstruction. All three patients had sufficient follow-up (mean of 21 months). Two patients were successfully treated with resolution of symptoms and obstruction by renal scan. The one failure alternatively had both persistent symptoms and continued obstruction on renal scan. For the laparoscopic procedures, the average length of stay was 2.5 days, blood loss was minimal and mean OR time was 390 minutes. For the robotic pyeloplasty, operative time was approximately 210 minutes with minimal blood loss $<25 \mathrm{ml}$ and a 2-day length of stay. Table 1 is a summary of our results. Our over-all success rate for the combined laparoscopic/robotic cohort was $67 \%$.

\section{Discussion}

Horseshoe kidneys were first described in the early autopsy studies of De Carpi in 1522, and in a more detailed fashion, the work of Morgagni in $1820 .{ }^{[8]}$ Since then, a great deal of data has been made available regarding this congenital anomaly. Although most cases are asymptomatic, horseshoe kidneys are associated with a higher incidence of UPJO and urolithiasis. ${ }^{[2,8,9]}$

In 1975, Pitts and associates published their experience with 170 patients with horseshoe kidneys over a 40 year period. ${ }^{[8]}$ Of these patients, $15 \%$ presented with UPJO. This series retrospectively noted an $80 \%$ success rate of Foley Y-V open pyeloplasty, 
although no follow-up was mentioned. In 1984, Das et al reported a success rate of open pyeloplasties in horseshoe kidneys of $55 \%(n=5) .{ }^{[10]}$

The first minimally invasive option for UPJO was an endoscopic intervention via retrograde or antegrade approach. In 1998, Jabbour et al published their experience with antegrade percutaneous endopyelotomy in horseshoe (4) and ectopic (5) kidneys. ${ }^{[2]}$ Of the horseshoe kidneys, $75 \%$ were noted to have clear improvement on follow-up IVP with reduction of hydronephrosis and rapid emptying of the renal pelvis and early visualization of the ureter. These three patients were also noted to be asymptomatic with a mean follow-up of 62 months. This success rate, although of a small sample, is identical to our endoscopic management of this condition.

Horseshoe kidneys, however, by definition contain anomalous blood supply and are prone to crossing vessels at the UPJ, and the presence of crossing vessels has been identified as a major risk in endopyelotomy failures (up to 87\%) for both normal and malformed kidneys. ${ }^{[11]}$ When we plan an endopyelotomy for a horseshoe kidney, we always perform an endoluminal ultrasound in the same setting in order to evaluate the UPJ. If we feel that the patient is a candidate for endopyelotomy, the endoluminal ultrasound allows us to plan the incision remote from any anomalous vessels that may be in the area. If, however, crossing vessels appear to be the etiology or the anatomy is otherwise unfavorable, we recommend a pyeloplasty. Two of the patients in our endopyelotomy cohort had crossing vessels, but these patients were considered poor surgical candidates for pyeloplasty and it was thought that endopyelotomy was their only option. All patients in the endopyelotomy group had not undergone any prior UPJ procedures. 
In 2004, Bove reported on the Johns Hopkins laparoscopic experience with UPJO in malformed kidneys. ${ }^{[12]}$ This series involved 5 horseshoe kidneys, 3 pelvic kidneys, a pancake kidney, a malrotated kidney and a duplicated collecting system. In this study, all patients showed clinical and/or radiographic success during follow-up of 32.6 and 21.3 months, respectively. Mean operative time for all anomalies was 195 minutes and mean estimated blood loss was $122 \mathrm{cc}$ with no patients needing blood transfusion. Average length of stay was 3.2 days. There were no intra-operative complications. Unfortunately these variables were not reported individually for horseshoe kidneys. In contrast, our data includes 2 patients with horseshoe kidneys who were treated laparoscopically for UPJO.

The choice of whether to perform a robotic or laparoscopic pyeloplasty at our institution is surgeon dependent. In 2005, Chammas and associates published a one-year follow-up of 3 patients with horseshoe kidneys who underwent robotic pyeloplasty. ${ }^{[13]}$ They found that all patients were both asymptomatic at one year and all had good renal function with improved drainage shown by IVU at 3 months. One patient had mild hydronephrosis, which resolved after one year. Mean operative time for this group of patients was 148.3 minutes. Two patients experienced complications in the post-operative period: obstructing urolithiasis and pyelonephritis.

We successfully treated one patient who had undergone a failed laser endopyelotomy in a horseshoe kidney with a robotic pyeloplasty. Operative time was 210 minutes, estimated blood loss was $25 \mathrm{~mL}$, and length of stay was 2 days. She remains symptom free and unobstructed on diuretic renogram after 2 years. All patients 
in our laparoscopic/robotic cohort had undergone a prior failed endopyelotomy, with 1 of these having been performed at our institution.

Table 2 documents a comparison of our results with those reported in the literature.

Our final recommendations for treatment of UPJO in a horseshoe kidney include careful delineation of the anomaly with either an endoluminal ultrasound or a CT angiogram. If the patient is considered a poor surgical candidate or if the transition point of the UPJO is not involving crossing vessels, endopyelotomy can be attempted with a reasonable success rate. In patients who have, however, undergone a failed endopyelotomy or if crossing vessels are the etiology of the UPJO, a laparoscopic/robotic pyeloplasty should be undertaken.

There are some shortcomings of the current study. First, it is retrospective and lends itself to the selection biases of such studies. Additionally, we were not able to obtain records in two of our patients who underwent endopyelotomy who had been lost to follow-up; this limits the conclusions that we can make for this procedure. Finally, our overall numbers are somewhat small and it is hard to draw definitive conclusions from the study, however we do feel that this is more representative of the rarity of this condition and our overall numbers represent, to our knowledge, the largest study to date that evaluates treatment of UPJO in a horseshoe kidney.

\section{Conclusion}

Horseshoe kidneys represent a unique challenge to UPJ reconstruction. In select patients, careful retrograde endoscopic endopyelotomy can result in moderate success 
and remains the least invasive option. Laparoscopic reconstruction with or without robotic assistance yields the highest success rate with long-term durability. Although data on the treatment of ureteropelvic junction obstruction via minimally invasive techniques in horseshoe kidneys is limited, our data suggests that endopyelotomy and laparoscopic/robotic pyeloplasty are all safe and effective options in well selected patients.

None of the authors contains any financial interest/arrangement that could be perceived as a real or apparent conflict of interest in the context of the subject of this article.

\section{References}

1. Hohenfellner, M., et al., Tumor in the horseshoe kidney: clinical implications and review of embryogenesis. J Urol, 1992. 147(4): p. 1098-102.

2. Jabbour, M.E., et al., Endopyelotomy for horseshoe and ectopic kidneys. J Urol, 1998. 160(3 Pt 1): p. 694-7.

3. Boatman, D.L., C.P. Kolln, and R.H. Flocks, Congenital anomalies associated with horseshoe kidney. J Urol, 1972. 107(2): p. 205-7.

4. Bauer, J.J., et al., Laparoscopic versus open pyeloplasty: assessment of objective and subjective outcome. J Urol, 1999. 162(3 Pt 1): p. 692-5.

5. Chen, R.N., R.G. Moore, and L.R. Kavoussi, Laparoscopic pyeloplasty. Indications, technique, and long-term outcome. Urol Clin North Am, 1998. 25(2): p. 323-30.

6. Weise, E.S. and H.N. Winfield, Robotic computer-assisted pyeloplasty versus conventional laparoscopic pyeloplasty. J Endourol, 2006. 20(10): p. 813-9.

7. Yanke, B.V., et al., Robot-assisted laparoscopic pyeloplasty: technical considerations and outcomes. J Endourol, 2008. 22(6): p. 1291-6.

8. $\quad$ Pitts, W.R., Jr. and E.C. Muecke, Horseshoe kidneys: a 40-year experience. J Urol, 1975. 113(6): p. 743-6.

9. Yohannes, P. and A.D. Smith, The endourological management of complications associated with horseshoe kidney. J Urol, 2002. 168(1): p. 5-8.

10. Das, S. and A.D. Amar, Ureteropelvic junction obstruction with associated renal anomalies. J Urol, 1984. 131(5): p. 872-4. 
11. Van Cangh, P.J., et al., Vessels around the ureteropelvic junction: significance and imaging by conventional radiology. J Endourol, 1996. 10(2): p. 111-9.

12. Bove, P., et al., Laparoscopic management of ureteropelvic junction obstruction in patients with upper urinary tract anomalies. J Urol, 2004. 171(1): p. 77-9.

13. Chammas, M., Jr., et al., Laparoscopic robotic-assisted management of pelviureteric junction obstruction in patients with horseshoe kidneys: technique and 1year follow-up. BJU Int, 2006. 97(3): p. 579-83.

14. Bellman, G.C. and R. Yamaguchi, Special considerations in endopyelotomy in a horseshoe kidney. Urology, 1996. 47(4): p. 582-5; discussion 585-6.

15. Koikawa, Y., et al., Percutaneous endopyelotomy for ureteropelvic junction obstruction in a horseshoe kidney. Scand J Urol Nephrol, 1996. 30(2): p. 145-7. 